\title{
Theoretical Study of the Electric Field Gradient in Silver Iodide*
}

\author{
G. Frantz**, R. Leiberich, and P. C. Schmidt \\ Institut für Physikalische Chemie, Petersenstr. 20, Technische Hochschule Darmstadt, \\ D-6100 Darmstadt, Germany
}

\section{T. P. Das}

Department of Physics, State University of New York at Albany, Albany, New York, 12222, USA

Z. Naturforsch. 47a, 182-188 (1992); received October 25, 1991

The electric field gradient (EFG) in hexagonal AgI at the iodine site is studied theoretically by three different procedures, namely the point charge model, the Hartree-Fock cluster approach, and the augmented plane wave band structure procedure. A comparison is made for the electronic charge distribution and the sizes of the various contributions to the EFG obtained by the different procedures. From the point charge model and the Hartree-Fock cluster approach one gets almost the same result for the EFG, $q=+0.743 \cdot 10^{21} \mathrm{~V} / \mathrm{m}^{2}$ and $+0.816 \cdot 10^{21} \mathrm{~V} / \mathrm{m}^{2}$ respectively. Only a rather preliminary result of $q=+0.393 \cdot 10^{21} \mathrm{~V} / \mathrm{m}^{2}$ is found by the band structure calculation to be compared with the experimental value of $q_{\exp }= \pm 0.415 \cdot 10^{21} \mathrm{~V} / \mathrm{m}^{2}$.

The confidence limits of the theoretical results are discussed, including possible ways to bridge the gap between the theoretical results by different methods and experiment. Both the cluster and the band structure calculations are shown to support an ionic model for hexagonal AgI with some overlap between the charge distributions on neighboring ions.

\section{Introduction}

Silver iodide crystallizes in four different structures [1]. Hyperfine properties have been studied experimentally by NMR technique in the hexagonal $\beta$-AgI phase [2], leading to a nuclear quadrupole coupling constant (QCC) of $7.91 \mathrm{MHz}$ for the ${ }^{127} \mathrm{I}$ nucleus [2]. Using the available quadrupole moment $Q\left({ }^{127} \mathrm{I}\right)=$ -0.789 barn [3], an experimental value of

$$
q_{\exp }= \pm 0.415 \cdot 10^{21} \mathrm{~V} / \mathrm{m}^{2}
$$

has been derived for the electric field gradient (EFG) at the iodine site from the measured QCC. This value is very small compared to the experimental values for covalently bonded iodine compounds, where the EFG at the iodine site is known to be [4] more than twenty times larger than in (1) for AgI.

The smallness of the experimental EFG could be understood qualitatively by a combination of sym-

* Presented at the XIth International Symposium on Nuclear Quadrupole Resonance Spectroscopy, London, U.K., July 15-19, 1991.

** Part of Dr.-Ing. thesis of Gerald Frantz, Technische Hochschule Darmstadt, D17.

Reprint requests to Dr. P. C. Schmidt, Institut für Physikalische Chemie, Technische Hochschule Darmstadt, Petersenstraße 20, D-6100 Darmstadt. metry considerations and the expected ionic nature of the bonding between $\mathrm{Ag}$ and I due to the large difference in electronegativity between $\mathrm{Ag}$ and I. The structure of $\beta$-AgI, shown in Fig. 1, is very close to the ideal wurzite structure [1] with a $c / a$ ratio of 1.635 and $z=0.3747 \pm 0.0015$, the corresponding values being 1.633 and 0.375 in the ideal case. The co-ordination of $\mathrm{Ag}^{+}$around $\mathrm{I}^{-}$ion is thus close to tetrahedral, leading

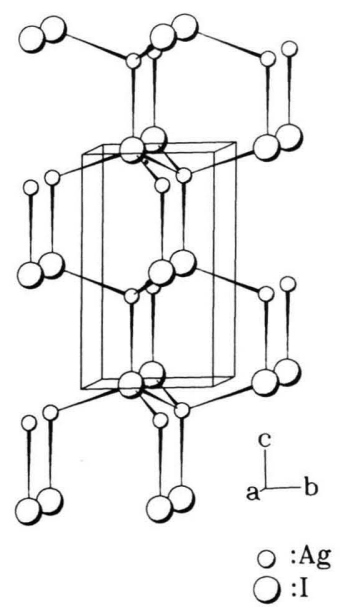

Fig. 1. The structure of hexagonal $\beta$-AgI, space group P6 ${ }_{3} \mathrm{mc}, a=459.2 \mathrm{pm}, c=751.0 \mathrm{pm}, c / a=1.635, z=0.3747$ \pm 0.0015 .

0932-0784 / $92 / 0100-0182 \$ 01.30 / 0$. - Please order a reprint rather than making your own copy. 
to an augmentation in the tendency towards a smaller QCC from the already expected small value from the ionic nature of Ag-I bonding.

The aim of the present paper is to analyze the EFG in $\beta$-AgI quantitatively by theoretical methods. For our investigations we have used three different values of $z$, namely the experimental mean value $z=0.3747$ and values 0.3732 and 0.3762 corresponding to the lower and upper limits of the experimental error range [1]. This has been done to study the sensitiveness of the theoretical results to the small but significant experimental uncertainty in $z$. This uncertainty is significant because the values of $z$ corresponding to the two limits of the experimental error range make the coordination of $\mathrm{Ag}$ around I depart from tetrahedral in oblate and prolate ways leading one to expect contributions to the EFG from the local distortion, which have opposite sign in the two cases.

The theoretical investigations are performed by three different procedures for comparison of the results by three procedures and also in the process to obtain a better assessment of the quantitative understanding of the origin of the EFG. First, in the pure ionic model, the overlap of the charge densities of the ions is neglected. The total EFG in this model is separated into the direct contribution $q_{\text {ext }}$ from the ionic charges of the lattice and the indirect contribution $q_{\text {ind }}$ from the polarization or induced effect produced by the point charges on the electron distribution of the ion containing the nucleus being considered. The ionic charges on the lattice are assumed to be charges totally external to the electron distribution of the central ion. The polarization effect is referred to in the literature as the Sternheimer antishielding effect and has been the subject of many investigations in the literature $[5,6]$.

The external term $q_{\text {ext }}$ can be written as a lattice sum over ionic charges (point charge model) [7],

$$
q_{\mathrm{ext}}=\sum_{v}^{\text {lat. }} Q_{v} \frac{3 z_{v}^{2}-r_{v}^{2}}{r_{v}^{5}}
$$

where $Q_{v}$ are the charges on the ions $v$ in the lattice, with radius vectors $\boldsymbol{r}_{v}$ with respect to the nucleus under study which is taken as the origin of the coordinate system.

The induced term $q_{\text {ind }}$ is included using the Sternheimer antishielding factor $\gamma_{\infty}[5,6]$, leading to

$$
q_{\text {ind }}=-\gamma_{\infty} q_{\text {ext }} .
$$

Since the antishielding factors which are obtained through perturbation calculations for ions in the presence of external charges, are usually known from earlier work [8], only the sum in (1) has to be evaluated to obtain the net field gradient, namely

$$
q=q_{\mathrm{ext}}+q_{\mathrm{ind}}=\left(1-\gamma_{\infty}\right) q_{\mathrm{ext}} .
$$

In a second procedure, $q$ is determined from the charge distribution obtained by a Hartree-FockRoothaan [9] molecular orbital (MO) calculation where the MO calculation is performed for a cluster of a certain number of ions and the influence of the rest of the lattice on the electronic states is described by point charges $[10,11]$. The size of the cluster is normally chosen to be as large as possible keeping in mind the considerations of practicability of computational effort involved. Within this procedure the oneelectron wave-functions $\psi_{i}(\boldsymbol{r})$ for all electrons in the cluster are determined first. The electronic charge density of the cluster is obtained from these wave-functions by the usual relation

$$
\varrho(\boldsymbol{r})=-e \sum_{i}\left|\psi_{i}(\boldsymbol{r})\right|^{2} .
$$

The contribution $q_{\text {clus }}$ of the cluster to the EFG is given by

$q_{\text {clus }}=\int \varrho(\boldsymbol{r}) \frac{3 z^{2}-r^{2}}{r^{5}} \mathrm{~d} \tau+\sum_{v}^{\text {clus. }} Z_{v} e \frac{3 z_{v}^{2}-r_{v}^{2}}{r_{v}^{5}}$,

where $Z_{v} e$ are the charges of the nuclei of the cluster. The contribution of the point charges on the lattice external to the cluster is again given by a lattice sum over these charges as in (2) with $Q_{v}$ referring to the effective charges on the ions, in contrast to the nuclear charges for ions in the cluster.

The one-electron states $\psi_{i}$ in (5) are expanded in terms of basis functions $\varphi_{v}$ centered at the nuclear positions in the form

$$
\psi_{i}=\sum_{v} c_{i, v} \varphi_{v},
$$

where the $\varphi_{v}$ are either primitive Gaussians or sets of contracted Gaussians [12], $c_{i, v}$ being the variation parameters that are determined through the HartreeFock-Roothaan procedure.

There are two differences between the point charge model and cluster approaches. First using $\gamma_{\infty}$ in the point charge model it is assumed that the antishielding effect on the electronic charge distribution of the ion containing the nucleus under consideration comes 
from totally external charges. In the cluster approach on the other hand, the influence of the net antishielding effect including the influence of the penetration [13] of the perturbing charges in the electronic charge distribution of the considered ion is taken into account in a first-principle manner, obviating the need for a parameter of the type $\gamma_{\infty}$ in (3). Equally important, in the cluster approach the covalent contribution to the chemical bond and its influence on the EFG is taken explicitly into consideration.

The cluster procedure simulates the infinite solid by a finite set of atoms and the influence of the potential of the rest of the ions in the lattice on the electrons within the cluster is approximated by the potential produced by point charges. The approximation of using a finite set of atoms in the cluster seems to be reasonable since we are interested in a localized property which can be considered as less sensitive to the infinite nature of the lattice than for instance, transport properties. Further, one usually studies the convergence of the properties such as the EFG being considered here with respect to the cluster size. This feature and the use of the potential from the ions in the lattice outside the cluster leads one to expect that the calculated results can be considered as representative of an infinite lattice. Nevertheless, we have made a comparison between the Hartree-Fock cluster procedure and the band structure procedure, in which periodic boundary conditions are used.

It should be pointed out, however, that while the cluster calculation uses the truly Hartree-Fock exchange potential, in the band structure approach we used the statistical exchange approximation [14] for practical reasons. A comparison between band structure and cluster procedures has been carried out before using two independent investigations $[10,15]$. In the band structure approach one can use the same expression for the EFG as in (6) for the cluster approach with $\varrho(\boldsymbol{r})$ given by (5) but with the $\psi_{i}(\boldsymbol{r})$ replaced by Bloch wave-functions $\psi_{k, n}(\boldsymbol{r}), \boldsymbol{k}$ referring to the wave-number and $n$ the band index. The sum over $i$ in (5) is now replaced by one over the occupied bands and the occupied $\boldsymbol{k}$ states in each band up to the Fermi-surface. When all the core and valence electrons are included in the band calculation, $Z_{v}$ in (6) again refers to the nuclear charge for the atoms in the metal. However, if for computational simplicity, only the valence electrons are used in the band calculation there are two modifications in the second term in (6), namely $Z_{v}$ is replaced by the effective charge $\left(Z_{v}-N_{v}\right)$ where $N_{v}$ is the number of core electrons and a factor $\left(1-\gamma_{\infty}^{\prime}\right)$ where $\gamma_{\infty}^{\prime}$ then corresponds to the Sternheimer antishielding factor for the core electrons.

In the present work the band structure calculation has been performed by the augmented plane wave (APW) method [16] in which the valence $5 \mathrm{~s}$ and $5 \mathrm{p}$ electrons of iodine and the $4 \mathrm{~d}$ and $5 \mathrm{~s}$ electrons of silver are handled as band electrons.

\section{Results and Discussion}

Next we present our results for the three models and compare them. From this comparison we shall attempt to access the relative accuracy of the three methods and their capabilities to provide the various contributions to the EFG at the ${ }^{127}$ I nucleus.

\section{Point Charge Model}

In order to study the dependence of the field gradient on the parameter $z$ we have evaluated the lattice sum in (2), using the formal charge +1 and -1 for $\mathrm{Ag}^{+}$and $\mathrm{I}^{-}$, for the mean $z$ value of the experimental measurement and the upper and lower limit. These values of $q_{\text {ext }}$, listed in Table 1 , are in excellent agree-

Table 1. Results for the EFG in $\beta$-AgI from ab initio and APW band structure calculations.

Lattice parameters [1] for $\beta$-AgI:

$a=459.2 \mathrm{pm}, c=751.0 \mathrm{pm}, c / a=1.635, z=0.3747 \pm 0.0015$ Space group: $\mathrm{P}_{3} \mathrm{mc}$

\begin{tabular}{|c|c|c|c|}
\hline \multirow[t]{2}{*}{$q / 10^{21} \mathrm{~V} / \mathrm{m}^{-2}$} & \multicolumn{3}{|l|}{$z$} \\
\hline & 0.3732 & 0.3747 & 0.3762 \\
\hline \multicolumn{4}{|c|}{ Point charge model } \\
\hline $\begin{array}{l}q_{\text {ext }}{ }^{\mathrm{a}} \\
q_{\text {ind }}{ } \\
q=q_{\text {ext }}+q_{\text {ind }}\end{array}$ & $\begin{array}{l}+0.0077 \\
+1.248 \\
+1.255\end{array}$ & $\begin{array}{l}+0.0045 \\
+0.738 \\
+0.743\end{array}$ & $\begin{array}{l}+0.0014 \\
+0.229 \\
+0.230\end{array}$ \\
\hline \multicolumn{4}{|c|}{$\mathrm{I}^{-}$cluster + point charges } \\
\hline $\begin{array}{l}q_{\text {ext }} \\
q_{\text {clus }} \\
q=q_{\text {ext }}+q_{\text {clus }}\end{array}$ & $\begin{array}{l}+0.0076 \\
+1.265 \\
+1.273\end{array}$ & $\begin{array}{l}+0.0044 \\
+0.812 \\
+0.816\end{array}$ & $\begin{array}{l}+0.0012 \\
+0.358 \\
+0.359\end{array}$ \\
\hline \multicolumn{4}{|c|}{$\left[\mathrm{Ag}_{4} \mathrm{I}\right]^{3+}$ cluster + point charges } \\
\hline $\begin{array}{l}q_{\text {ext }} \\
q_{\text {clus }} \\
q=q_{\text {ext }}+q_{\text {clus }}\end{array}$ & $\begin{array}{l}+0.0037 \\
+1.006 \\
+1.010\end{array}$ & $\begin{array}{l}+0.0040 \\
+0.841 \\
+0.845\end{array}$ & $\begin{array}{l}+0.0043 \\
+0.705 \\
+0.709\end{array}$ \\
\hline \multicolumn{2}{|c|}{ APW band structure calculation } & $\begin{array}{l}+0.393 \\
\pm 0.415\end{array}$ & \\
\hline
\end{tabular}

${ }^{\mathrm{a}}$ Ref. [17]. ${ }^{\mathrm{b}}$ Using $\gamma_{\infty}=-162$ [8].

c Obtained from [2] using $Q\left({ }^{127} \mathrm{I}\right)=-0.789$ barn [3]. 
ment with those obtained in an earlier work by Sholl [17]. The values of $q_{\text {ind }}$, (3), corresponding to the three different $q_{\text {ext }}$ values are also listed in Table 1 for the antishielding factor of $\gamma_{\infty}=-162$ [8]. In view of the very large values of $q_{\text {ind }}$ compared to $q_{\text {ext }}, q$ is almost entirely determined by the latter.

These results for the point charge model have two important features. First there is a very sensitive deviation of $q$ on the parameter $z$. Second even though the dependence from tetrahedral symmetry of the nearest neighbors around the $\mathrm{I}^{-}$ion undergoes a change from prolate to oblate character in the error range in $z$, the total $q$ does not change sign. This latter result can be understood by comparing the value of $q$ in Table 1 with the values from the nearest neighbors, namely $+0.624 ;+0.059 ;-0.510$ in units of $10^{21} \mathrm{~V} / \mathrm{m}^{2}$. This contribution, while very sizable, is smaller in magnitude than the contribution of the rest of the lattice.

Among the main criticism that can be leveled against the simple point charge estimate in Table 1 are that a) they do not take account of the extension of the $\mathrm{I}^{-}$charge distribution beyond the nearest neighbors, that b) solid state effects on the $\gamma_{\infty}$ used are included in a somewhat approximate manner through the Watson sphere model [8] and that c) they do not include the influence of overlap and binding effects between $\mathrm{I}^{-}$ ion and its neighboring $\mathrm{Ag}^{+}$ions.

To study the influence of the first two effects we have carried out a self-consistent calculation for $\mathrm{I}^{-}$in the presence of both its neighboring $\mathrm{Ag}^{+}$ions considered as point charges as well as the rest of the ions in the lattice.

For practicability reasons we have included up to 755 neighbors of the $\mathrm{I}^{-}$ion involving shell wise summation up to 189 unit cells. In including the influence of the point charges on the potential, experienced by the electrons on the $\mathrm{I}^{-}$ion, we have grouped these ions in ensembles in which the lowest non-vanishing moment is a octupole [18] in a manner similar to the Evjen procedure [19] for the Madelung constant. This procedure is somewhat different than that in other recent cluster calculations of EFG's in ionic crystals $[10,11]$. The validity of the procedure is supported by the very close agreement in the $q_{\text {ext }}$ from this procedures and essentially infinite lattice summation in the point charge model and also the closeness of the calculated Madelung constant for $\mathrm{I}^{-}$ion of 1.639 to the correct value of 1.642 [20].

The net $q$ from this calculation for the different values of $z$, given in Table 1, are not very different from those for the pure point charge model. The basis set dependence of $q_{\text {theor }}$ is discussed below for the cluster calculation. The value of $q$ for the mean value of $z$ is about twice as large as experiment. However, the value of the lower extreme of the error range in $z$ is already lower than experiment, indicating the need of a tightening of the experimental error range of $z$.

\section{Cluster Approach}

We have followed the two types of point charge model calculation just discussed by an all-electron self-consistent-field Hartree-Fock cluster calculation including the potentials of the ions outside the cluster by the point charge model. The cluster used involved an $\mathrm{I}^{-}$ion in the center surrounded by four immediate $\mathrm{Ag}^{+}$neighbors. Now the number of external charges is 751 ions decreased by four ions used in the calculation involving only an $\mathrm{I}^{-}$ion in the center just discussed.

The calculations were carried out using Gaussian basis sets and the quantum chemistry program HONDO which is a part of the program package MOTECC [21]. For the central ion $\mathrm{I}^{-}$two basis sets were used namely $(15 \mathrm{~s}, 11 \mathrm{p}, 6 \mathrm{~d})$ and $(18 \mathrm{~s}, 14 \mathrm{p}, 8 \mathrm{~d})$ which are contracted to $[10 \mathrm{~s}, 8 \mathrm{p}, 4 \mathrm{~d}]$ and $[16 \mathrm{~s}, 12 \mathrm{p}$, $8 \mathrm{~d}]$ [22]. These basis sets correspond to the neutral iodine atom. This choice is expected to be a satisfactory one because of previous experience with $\mathrm{O}^{2-}$ in $\mathrm{ZnO}$ [23], where the results using basis functions for neutral oxygen were found to agree well with those using $\mathrm{O}^{2-}$ basis functions calculated in a Watson sphere. For silver ions a minimal basis set $(15 \mathrm{~s}, 9 \mathrm{p}, 6 \mathrm{~d})$ contracted to [ $5 \mathrm{~s}, 3 \mathrm{p}, 2 \mathrm{~d}]$ [24] was used for the sake of economy of computational effort. In Table 2 the calculated EFG for both basis sets are shown. We see that the results for both basis sets differ by approximately $10 \%$. As a function of the parameter $z$ both basis sets give the same trends.

In Table 1 we have listed the contribution of the EFG of the cluster $q_{\text {clus }}$ and outside $q_{\text {ext }}$, the former for the more flexible basis set on iodine. The external contribution $q_{\text {ext }}$ being different from that for the point charge calculation because the latter includes the contribution from the nearest neighbors.

In any case $q_{\text {ext }}$ is very small compared to the contribution $q_{\text {clus }}$ within the cluster. However the sizable antishielding effects are included in $q_{\text {clus }}$ through the polarization of the electron distribution in the cluster. 
The net field gradient $q$ has the following features: first as in the case of the two point charge type calculations there is a sensitive dependence on the parameter $z$. However, the variation is somewhat less pronounced than in the other two cases. Second, except in the case of the mean value $z$ corresponding to near tetrahedral co-ordination of iodine there is a significant change from going from the $\mathrm{I}^{-}$cluster investigation to the $\left[\mathrm{Ag}_{4} \mathrm{I}\right]^{3+}$ cluster. These changes reflect the influence associated with the overlap of the charge distribution of the $\mathrm{I}^{-}$and $\mathrm{Ag}^{+}$ions and associated with a charge transfer covalency effect. Since the total charges we obtained for $\mathrm{I}^{-}$and $\mathrm{Ag}^{+}$ions using the Mulliken approximation [25] leads to effective charges of $-0.96792 \mathrm{e}$ for the $\mathrm{I}^{-}$and $+0.99144 \mathrm{e}$ for the $\mathrm{Ag}^{+}$ ions it appears that charge covalency effects might be less important relative to the overlap effect. Lastly, in making comparison with the experiment one notices that the value of $q$ for the mean value of $z$ is about twice as large as the expected one, with the agreement getting better for upper extreme $z$ value.

In trying to understand the difference between theory and experiment one could consider a number of sources. First, it is possible that the basis sets which have been employed are not large enough as one sees from the variation between the values of $q$ for the two basis sets, see Table 2. It would be helpful to explore this situation further including larger basis sets in the future since closer agreement with the experiment is found for other ionic crystals [11,23]. Secondly, since the $\mathrm{I}^{-}$and $\mathrm{Ag}^{+}$ions are fairly sizable ions it may be that the use of five ion clusters is not sufficiently large enough in contrast to the case of $\mathrm{ZnO}$ [23] where such a cluster was found to be adequate. This is another feature to be explored in the future remembering that

Table 2. Comparison of the EFG values calculated with different basis sets and different $z$ parameters.

\begin{tabular}{|c|c|c|c|c|}
\hline \multirow{2}{*}{$\begin{array}{l}q_{\text {clus }} / \\
10^{21} \mathrm{~V} / \mathrm{m}^{-2}\end{array}$} & \multirow{2}{*}{$\begin{array}{l}\text { Basis } \\
\text { set }\end{array}$} & \multicolumn{3}{|l|}{$z$} \\
\hline & & 0.3732 & 0.3747 & 0.3762 \\
\hline $\begin{array}{l}\mathrm{I}^{-} \text {cluster } \\
+ \text { point charges }\end{array}$ & $\begin{array}{l}\# 1^{\mathrm{a}} \\
\# 2^{\mathrm{b}}\end{array}$ & $\begin{array}{l}+1.409 \\
+1.273\end{array}$ & $\begin{array}{l}+0.9 \\
+0.8\end{array}$ & $\begin{array}{l}+0.457 \\
+0.359\end{array}$ \\
\hline $\begin{array}{l}{\left[\mathrm{Ag}_{4} \mathrm{I}\right]^{3+} \text { cluster }} \\
+ \text { point charges }\end{array}$ & $\begin{array}{l}\# 1^{\mathrm{a}, \mathrm{c}} \\
\# 2^{\mathrm{b}, \mathrm{c}}\end{array}$ & $\begin{array}{l}+1.108 \\
+1.010\end{array}$ & $\begin{array}{l}+0 \\
+0\end{array}$ & + \\
\hline \multicolumn{5}{|c|}{$\begin{array}{l}\text { a Using a Gaussian basis set [22] }(15 \mathrm{~s}, 11 \mathrm{p}, 6 \mathrm{~d}) \text { with a con- } \\
\text { traction scheme [10s, } 8 \mathrm{p}, 4 \mathrm{~d}] \text { for } \mathrm{I} \text {. } \\
\text { b Using a Gaussian basis set [22] }(18 \mathrm{~s}, 14 \mathrm{p}, 8 \mathrm{~d}) \text { with a con- } \\
\text { traction scheme [16s, 12p, 8d] for I. } \\
\text { c Using a Gaussian basis set [24] }(15 \mathrm{~s}, 9 \mathrm{p}, 6 \mathrm{~d}) \text { with a con- }\end{array}$} \\
\hline
\end{tabular}

an all electron calculation for larger clusters and larger basis sets could not be performed in the present work because of computer time and storage limits. In addition to this, relativistic and many body effects should also be explored.

\section{Band Structure Results}

As mentioned earlier, we have carried out a band structure calculation for this system by the conventional muffin-tin APW procedure [16]. This calculation is rather time consuming because of convergence problems associated with the localized electron distribution in the ionic crystal system and associated narrow bands in $\boldsymbol{k}$-space in contrast to the situation in metals. Due to computer time limitations we could not perform a full potential calculation [26] and we could not study the EFG as a function of the $z$ parameter as in the case of the cluster calculation. The difficulties manifest themselves in terms of slow convergence with respect to the basis sets in the $\boldsymbol{k}$-space and angular momentum ( $l$ ) components inside the muffintins. Typically one requires 200 functions in $\boldsymbol{k}$-space and one has to go up to $(l=12)$ in angular momentum. Additionally the demands on the accuracy in solving the secular equation in the APW procedure are much more severe, the determination of the smallest diagonal element $D_{0}$ (see [27]) requires a value for $D_{0}$ which is about eight orders of magnitude smaller than the next largest diagonal element $D_{v}$. Due to computer time limitations associated with these convergence and accuracy problems we could only scan a maximum of $28 \boldsymbol{k}$-points in the irreducible part (1/24 th) of the Brillouin zone. The convergence with respect to the scanning process could not be studied. Therefore the value of the $\mathrm{EFG},+0.393 \cdot 10^{21} \mathrm{~V} / \mathrm{m}^{2}$, obtained for the choice of the mean value of the $z$ parameter appears to be only a preliminary band structure result. The value is in surprisingly good agreement with the experimental value of $\pm 0.415 \cdot 10^{21} \mathrm{~V} / \mathrm{m}^{2}$. However, in view of the fact that there are uncertainties in this result due to the various convergence and accuracy effects discussed and the fact that the variation with the $z$ parameter has not been studied, the status of the agreement between experiment and band structure theory is somewhat uncertain. It would be desirable in the future to study the variation with $z$ and also carry out calculations without the muffin-tin approximation. However it should be pointed out that there is a 


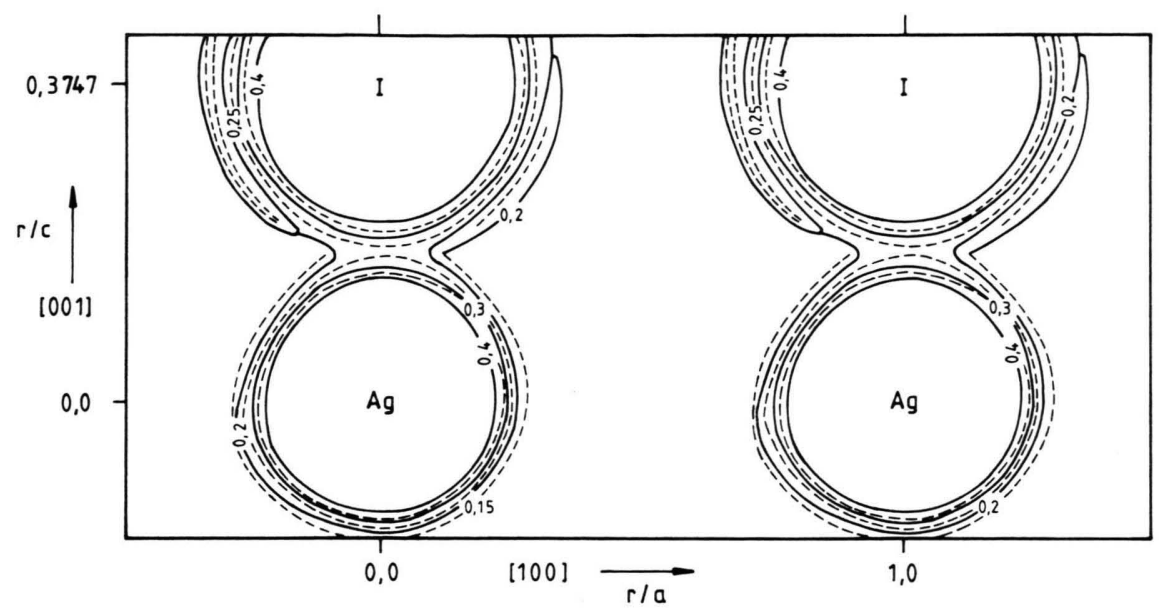

Fig. 2. The valence electron densities of $\beta$-AgI in the (010) plane. The cut-off of the contour plot is $0.4 \mathrm{e} \AA^{-3}$.

good agreement in some aspects between band structure and cluster calculation which influences the EFG; thus both calculations lead to almost complete ionic charge for iodine and silver. In the band structure calculation this conclusion is made on the fact that the $5 \mathrm{~s}$ like states of silver are found to be completely empty since they lie distinctly above the Fermi energy. Secondly, the charge density contour plot shown in Fig. 2 is indicative of the picture of overlapping ionic spheres, a result which is also suggested by the Mulliken population analysis [25] for the cluster calculation, see above. Lastly, from a density of state analysis one finds peaks in the density of state at similar energies where there is bunching up of energy levels from cluster calculations, with nearly identical admixture of iodine $5 \mathrm{p}$ and silver $4 \mathrm{~d}$ character for the states in both calculations.

\section{Conclusions}

The charge distribution in $\beta$-AgI has been shown to be described quite satisfactorily by the ionic model. This is also demonstrated by the fact that the results

[1] G. Burley, J. Chem. Phys. 38, 2807 (1963).

[2] S. L. Segel, J. A. Walter, and J. Troup, Phys. Stat. Sol. 31, K 43 (1969); D. Brinkmann and W. Freudenreich, Z. Kristallogr. 143, 67 (1976).

[3] M. Lederer and V. S. Shirley, Table of Isotops, $7^{\text {th }}$ Ed., Wiley Interscience, 1978.

[4] See e.g. H. Dreizler, Z. Naturforsch. 47a, 342 (1991).

[5] H. Foley, R. Sternheimer, and D. Tycko. Phys. Rev. 93, 734 (1954); T. P. Das and R. Bersohn, Phys. Rev. 102, for the electric field-gradient by a self-consistent treatment of an $\mathrm{I}^{-}$ion in the field of the point charges in the lattice is quite close to that from a cluster calculation involving $\mathrm{I}^{-}$and its nearest $\mathrm{Ag}^{+}$neighbors in the potential due to the charges in the rest of the lattice. There is, however, some difference between the two which appears to be a consequence of the overlap effect between the $\mathrm{I}^{-}$and $\mathrm{Ag}^{+}$neighbors. While there is reasonable overall agreement between theoretical and experimental results for the electric field gradient at the ${ }^{127}$ I nucleus, there are some quantitative differences between theoretical results and experiment. To improve the quantitative agreement in future investigations a larger numerical demand for the theoretical study as well as a careful redetermination of the crystal structure of $\beta$-AgI is needed.

\section{Acknowledgements}

We gratefully thank the "Fonds der Chemischen Industrie" for support of this work. The collaborative effort for this work was supported by NATO Research Grant No. 487/88.

733 (1956); R. Sternheimer, Z. Naturforsch. 41 a, 24 (1986).

[6] T. P. Das and P. C. Schmidt, Z. Naturforsch. 41 a, 47 (1986).

[7] R. Bersohn, J. Chem. Phys. 29, 326 (1958).

[8] P. C. Schmidt, K. D. Sen, T. B. Das, and Al. Weiss, Phys. Rev. B 22, 4167 (1980).

[9] C. C. J. Roothaan, Rev. Mod. Phys. 23, 69 (1951); G. G. Hall, Proc. Roy. Soc. London A 205, 541 (1951), W. 
Pauli, Z. Physik 31, 765 (1925); V. Fock, Z. Physik 61, 126 (1930); J. C. Slater, Phys. Rev. 35, 210 (1930).

[10] P. C. Kelires, K. C. Mishra, and T. P. Das, Hyperfine Interact. 34, 289 (1987).

[11] P. C. Kelires and T. P. Das, Hyperfine Interact. 34, 285 (1987); J. Stein, S. B. Sulaiman, N. Sahoo, and T. P. Das, Hyperfine Interact. 60, 849 (1991); H. H. Klauss, N. Sahoo, P. C. Kelires, T. P. Das, W. Potzel, M. Kalvius, M. Frank, and W. Kreische, Hyperfine Interact. 60, 853 (1990).

[12] E. Clementi and D. R. Davis, J. Chem. Phys. 45, 2593 (1966); J. Computational Phys. 2, 223 (1967).

[13] A. C. Beri, R. M. Sternheimer, T. Lee, and T. P. Das, Phys. Rev. B 28, 2335 (1983).

[14] P. Hohenberg and W. Kohn, Phys. Rev. 136, B 864 (1964); W. Kohn and L. J. Sham, Phys. Rev. 140, A 1133 (1965).

[15] P. Blaha, K. Schwarz, and P. Herzig, Phys. Rev. Lett. 54, 1192 (1985).

[16] J. S. Slater, Phys. Rev. 51, 846 (1937); J. C. Slater, Adv. Quantum Chem. 1, 35 (1964); T. L. Loucks, Augmented Plane Wave Method, W. A. Benjamin, New York 1967; L. F. Mattheiss, J. H. Wood, and A. C. Switendick, Methods Comput. Phys. 8, 64 (1968).

[17] C. A. Sholl, Proc. Phys. Soc. London 87, 897 (1966).

[18] H. Cocker, J. Phys. Chem. 87, 2512 (1983); F. Seitz and D. Turnbull, Solid State Physics, Vol. 16, Academic Press, New York 1964.
[19] H. M. Evjen, Phys. Rev. 39, 675 (1932).

[20] R. C. Weast and M. J. Astle, CRC Handbook of Chemistry and Physics $63^{\text {rd }}$ Ed, CRC Press Inc., Boca Raton, Florida, 1982.

[21] HONDO 8, M. Dupuis, J. D. Watts, H. O. Villar, and G. J. B. Hurst, Program no. 544, Quantum Chemistry Program Exchange, India University, Bloomington, India.

[22] R. Poirier, R. Kari, and J. G. Csizmadia, Handbook of Gaussian basis sets, Elsevier Amsterdam, 1985; A. Stomberg, O. Gropen, and U. Wahlgren, J. Comput. Chem. 4, 181 (1983); S. Huzinaga, J. Chem. Phys. 71, 1980 (1979).

[23] D. W. Mitchell, S. B. Sulaiman, N. Sahoo, T. P. Das, W. Potzel, and G. M. Kalvius, Phys. Rev. B, in press.

[24] R. Poirier, R. Kari, and J. G. Csizmadia, Handbook of Gaussian basis sets, Elsevier, Amsterdam, 1985; Y. Sakai, H. Tatewaki, and S. Huzinaga, J. Comput. Chem. 3, 6 (1982).

[25] R. S. Mulliken, J. Chem. Phys. 23, 1833, 1841, 2338, 2343 (1955).

[26] P. Blaha and K. Schwarz, Z. Naturforsch. 47a, 197 (1991).

[27] L. F. Mattheiss, J. H. Wood, and A. C. Switendick, In: Methods in Computational Physics, Vol. 8, ed. by B. Alder, Academic Press, New York 1968, p.109. 